\title{
A 16-Channel Reconfigurable OCDMA/DWDM System Using Continuous Phase-Shift SSFBGs
}

\author{
Chun Tian, Zhaowei Zhang, Morten Ibsen, Periklis Petropoulos, and David J. Richardson
}

\begin{abstract}
We demonstrate a reconfigurable 16-channel optical code-division multiple access (OCDMA)/dense wavelength division multiplexing (DWDM) system $(4$ OCDMA $\times 4$ DWDM $\times$ $625 \mathrm{Mb} / \mathrm{s}$ ) based on novel 31-chip, $40 \mathrm{Gchip} / \mathrm{s}$ quaternary phase coding gratings operating at a channel spacing of just $50 \mathrm{GHz}$. The system performance is studied for cases of both fixed and code-reconfigurable decoders. Error-free performance is achieved in both cases and for all 16 channels.
\end{abstract}

Index Terms-Fiber Bragg grating, optical code-division multiple access, optical fiber communication, wavelength division multiplexing (WDM).

\section{INTRODUCTION}

$\mathbf{O}$ PTICAL code-division multiple access (OCDMA) is currently attracting a great deal of research interest since it presents a variety of attractive features for future optical networks. These include the possibility of asynchronous operation, flexible bandwidth management, improved system security, and the potential for higher network scalability. Central to the successful practical implementation of OCDMA concepts is the development of practical code generation and recognition techniques that support multiple users at a reasonable spectral efficiency and which at the same time offer user code reconfiguration and the potential for low cost. Phase-encoded directsequence OCDMA seems a particularly promising approach according to which individual data bits are labeled directly in the time domain with some (fast) phase modulation profile according to a specific code sequence. The use of superstructured fiber Bragg grating (SSFBG) technology [1], [2] represents an attractive means to perform the phase encoding/decoding. Relative to other coding approaches, SSFBGs offer advantages in terms of fiber compatibility, ease and cost of manufacture, the ability to support long phase and amplitude code sequences, and the inherent wavelength selectivity that make the devices highly suitable for use within wavelength division multiplexing (WDM) systems. To demonstrate these benefits, we previously considered a 4-channel ( 2 OCDM $\times 2 \mathrm{WDM}$ at 4-nm interval) system, using both 63-chip 160-Gchip/s BPSK SSFBGs and 255-chip 320-Gchip/s QPSK SSFBGs [3]. We also demonstrated a 4 $\mathrm{OCDM} \times 4 \mathrm{WDM} \times 311 \mathrm{Mb} / \mathrm{s}$ OCDMA system operating on a 100-GHz grid using fixed 16-chip, 20-Gchip/s, quaternary phase coding SSFBGs [4]. These experiments verified that SSFBGs

Manuscript received December 20, 2006; revised March 21, 2007. This work was supported in part by the FP6 Network of Excellence E-Photon/ONe IST027497.

The authors are with the Optoelectronics Research Centre, University of Southampton, Southampton SO17 1BJ, U.K. (e-mail: cht@orc.soton.ac.uk; zhz@orc.soton.ac.uk; mi@orc.soton.ac.uk; pp@orc.soton.ac.uk; djr@orc. soton.ac.uk).

Digital Object Identifier 10.1109/JSTQE.2007.897668 offer a practical and flexible approach to multiuser operation with different coding schemes, data rate, and wavelength channels. One limitation of the basic SSFBG approach is that the codes are fixed during the writing process, and cannot be readily changed by the user. Consequently, to address this issue, we developed a code-reconfigurable grating device, in which the phase shifts are produced by a thermally induced refractive index variation along an otherwise uniform FBG [5], [6].

We have investigated the thermally induced phase-shift distribution along the grating using an electroabsorption modulatorbased frequency-resolved optical gating (EAM-FROG) technique [7] and revealed that the thermally induced phase shift is, in fact, distributed over a length of a few millimeters with a nearly hyperbolic profile [8]. Another important feature of the reconfigurable grating is that it has a narrower spectrum compared to the normal discrete phase-shift SSFBGs. Despite the thermally induced distributed phase shift, the codereconfigurable device has proven to be compatible with the use of normal discrete phase-shift SSFBGs [9]. Furthermore, we recently proposed a fixed-coded continuous phase-shift SSFBG [10]. This novel grating is designed to have a similar distributed phase distribution as the reconfigurable grating, thereby achieving a similarly narrow spectral bandwidth and a better match to the reconfigurable grating. As opposed to previous demonstrations of continuous phase-shift gratings [11], [12], in our case the continuous phase shifts are implemented directly in the grating writing process. Our preliminary study based on 16chip gratings indicated that the continuous phase-shift SSFBGs are more tolerant to broader input coding pulses than discrete phase-shift SSFBGs thereby having the potential to reduce the overall system bandwidth, complexity, and associated cost [10].

In this paper, we report the use of these new technologies to demonstrate a 16-channel, reconfigurable OCDMA/DWDM system operating with improved spectral efficiency on a $50-\mathrm{GHz}$ DWDM-compatible grid. The paper is organized as follows: Section II describes and compares the performance of the fixed and the code-reconfigurable gratings used in our experiments. The configuration and settings of our DWDM transmitter are described in Section III, and our system experiments are given in Section IV. We repeated our experiments using both a fixed and a code-reconfigurable decoder, and found that error-free performance could be achieved in all cases. The paper closes in Section V with some conclusions on this work.

\section{Continuous Phase-Shift SSFBGs AND CODE-RECONFIGURABLE GRATING}

In the experiments described in this paper, we have used encoding/decoding devices based on two different fiber-grating 
technologies. Fixed SSFBG encoders/decoders have been fabricated incorporating continuous phase shifts (in contrast to discrete phase shifts, as described, e.g., in [1]). We have also employed a code-reconfigurable device for decoding based on the thermo-optic effect that allowed us to dynamically address the decoded sequence. All gratings carry 31-chip, 40-Gchip/s quaternary phase codes chosen from the FamilyA code sequences that offer optimal correlation characteristics [13]. The chip length for these gratings is $2.5 \mathrm{~mm}$ (25-ps chip duration), resulting in a total grating length of $77.5 \mathrm{~mm}$. The four codes used in this experiment are:

$$
\begin{aligned}
& \mathrm{F} 1=\left[\begin{array}{l}
3202330331212322021201213023313
\end{array}\right] \\
& \mathrm{F} 2=\left[\begin{array}{l}
3121132121022032210130213113022
\end{array}\right] \\
& \mathrm{F} 3=\left[\begin{array}{l}
2323022012230310231331022132331
\end{array}\right] \\
& \mathrm{F} 4=\left[\begin{array}{l}
3312223301310101222030212112211
\end{array}\right]
\end{aligned}
$$

In this paper, the individual gratings used are labeled according to the following rules: The first letter defines the grating type; "F" stands for a fixed-coded continuous phase-shift SSFBG, "R" stands for the reconfigurable grating. The first number (1-4) identifies the four different codes. The second number (1-4) indicates the wavelength. Asterisk $(*)$ is used to indicate whether the grating is for decoding rather than coding a particular channel. For example, "F32" means fixed-coded grating with code 3 , at $\lambda 2$, and R32* the corresponding reconfigurable decoder grating.

A description of the different types of gratings is presented in the following paragraphs. The code-reconfigurable grating is described first, since its characteristics were used as the basis for the design of the continuous phase-shift SSFBGs.

\section{A. Code-Reconfigurable Grating}

Fig. 1(a) illustrates the structure of the reconfigurable grating [5], [6]. A 77.5-mm uniform FBG is mounted on a fiber stretcher to allow tuning of the grating Bragg wavelength. There are 30 tungsten wires distributed at $2.5-\mathrm{mm}$ intervals along the FBG. Each of the wires can be individually temperature tuned to induce the desired phase code distribution along the grating length. The operation of this device is described in [6], where it is shown that it is possible to tune from one code to another by simple reassignment of the current flowing through each of the tungsten wires (and, hence, their temperatures). The time required to tune between codes is $\sim 2 \mathrm{~s}$. The device is mounted on a stretching rig, which also allows for fine tuning of its central wavelength. Fig. 1(b) shows the reflectivity spectra of the device when it is programmed with four different codes (R13*, R23*, R33*, R43*, respectively) and tuned to wavelength $\lambda_{3}$ $(1559.80 \mathrm{~nm})$.

Using the EAM-FROG technique, we were able to measure the extent of the thermally induced phase distributions within the reconfigurable grating [8]. We subsequently used this information to design the phase distribution associated with each chip transition within the fixed continuous phase-shift SSFBGs, and to ensure optimal matching performance between the two types of devices.

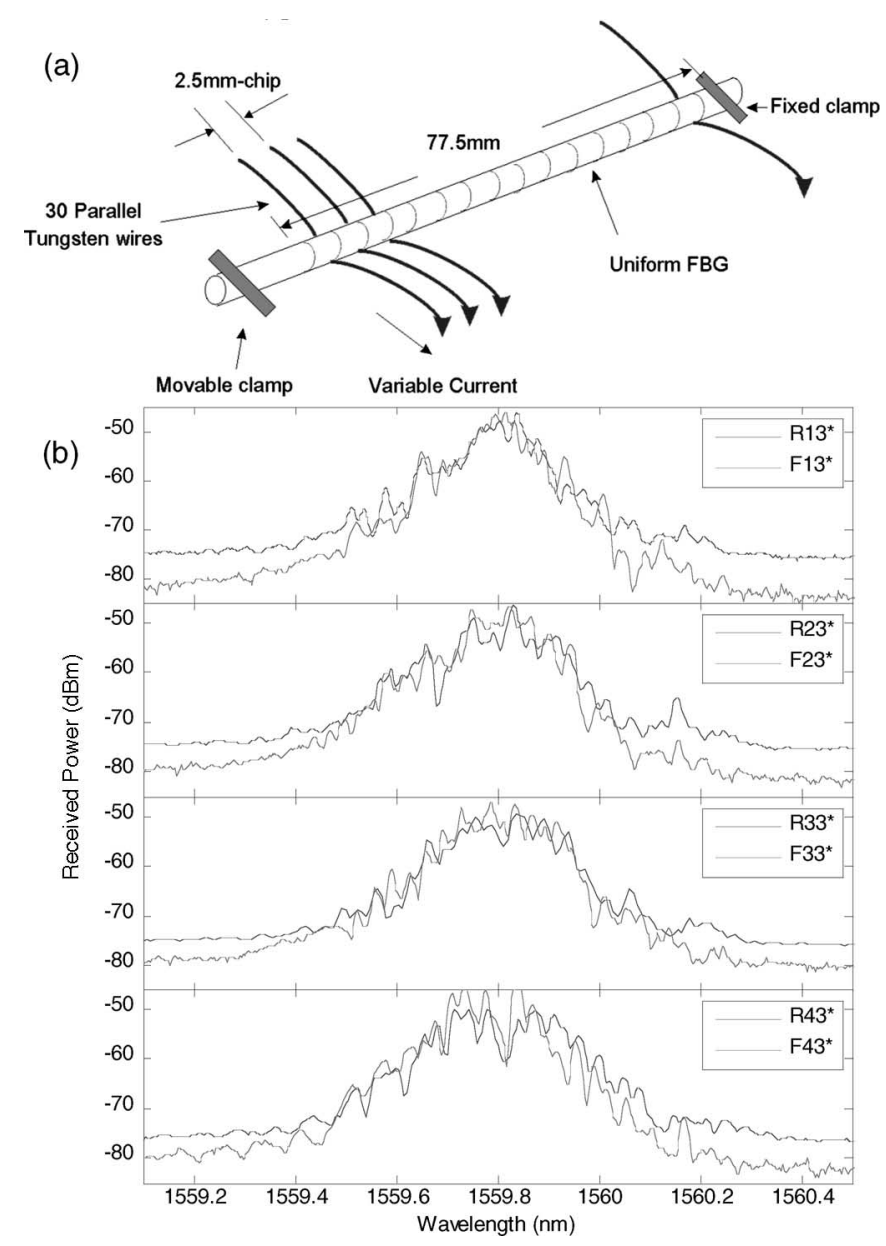

Fig. 1. (a) Structure of the 31-chip reconfigurable grating. (b) Reflectivity spectra of the reconfigurable grating (solid line) and the continuous phase-shift SSFBGs (dash line) for four different codes.

\section{B. Continuous Phase-Shift SSFBGs}

Fig. 2(a) shows the effective refractive index distributions corresponding to a single phase-shift $0.5 \pi, 1.0 \pi, 1.5 \pi$ in the quaternary OCDMA codes of the fixed continuous phase-shift SSFBGs. As mentioned previously, this was designed using the measured characteristics of the code-reconfigurable device as a basis. The matched continuous phase distribution profiles have been shown to provide better correlation performance than the combination of discrete and continuous phase-shift codes. Using distributed phase shifts in the SSFBG structure also allows the grating bandwidth to be reduced, which was critical in our OCDMA/DWDM experiments in order to ensure efficient bandwidth utilization. The phase distribution profiles for the discrete phase-shift SSFBG and the continuous phase-shift SSFBG with the same code F2 are illustrated in Fig. 2(b). Fig. 3 shows the measured and simulated reflectivity spectrum of grating F22. For comparison, we also plot the simulated spectrum of a grating with the same code and strength as grating F22 but with a discrete rather than continuous chip-phase transition. It can be readily seen that the continuous phase-shift SSFBG has a narrower 20 -dB bandwidth $(\sim 0.45 \mathrm{~nm})$ and no unwanted side lobes, 

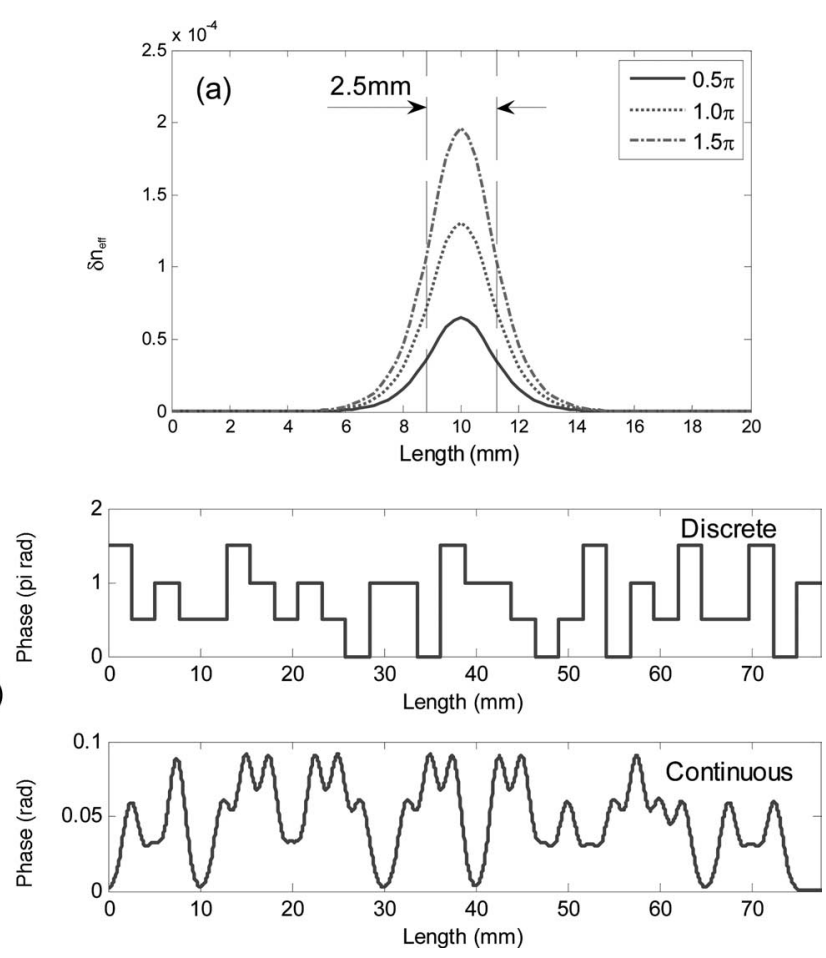

Fig. 2. (a) Effective refractive index distributions of a single phase-shift within the continuous phase-shift SSFBGs. (b) Phase distribution profile for the discrete phase-shift SSFBG and the continuous phase-shift SSFBG with the same code F2.

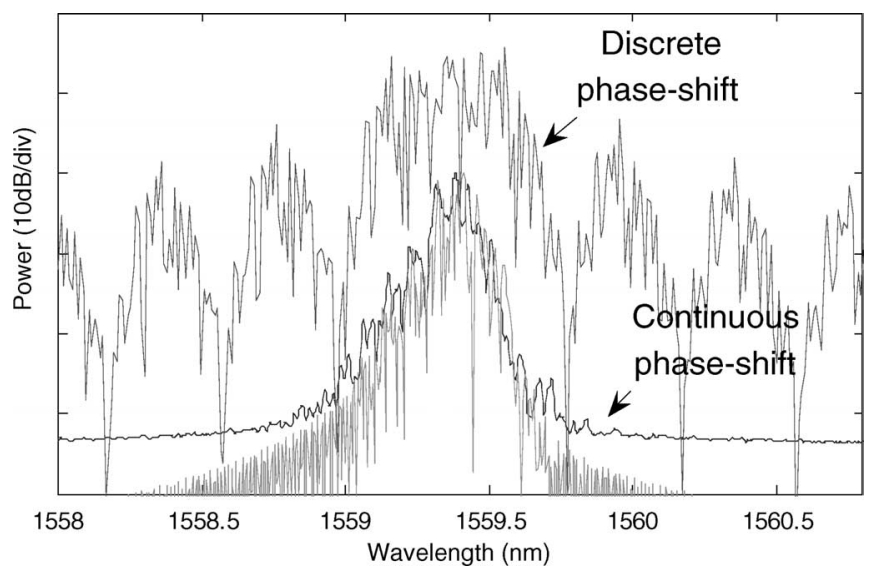

Fig. 3. Reflectivity spectra of grating F22 (solid line, measurement; dotted line, simulation; dashed line, simulation of a discrete phase-shift grating, upshifted by $15 \mathrm{~dB}$ to avoid overlap with the continuous phase-shift data).

allowing for much closer wavelength channel spacings than were previously possible using discrete phase-shift gratings.

In order to allow a comparison between the performance of the continuous phase-shift SSFBG and the code-reconfigurable grating, we have superimposed the reflectivity spectra of the two devices in Fig. 1(b). The good agreement between the reflectivity spectra of the continuous fixed phase-shift SSFBGs and that of the reconfigurable grating confirms the results of our previous measurements of the thermally induced phase distributions within the reconfigurable grating, and shows that the reconfigurable grating works as expected.

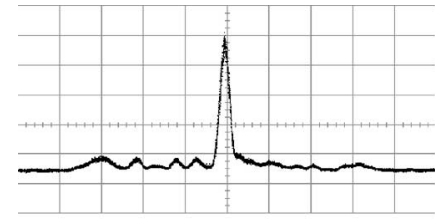

(a)

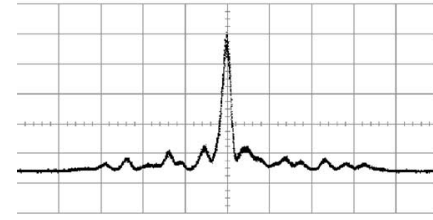

(c)

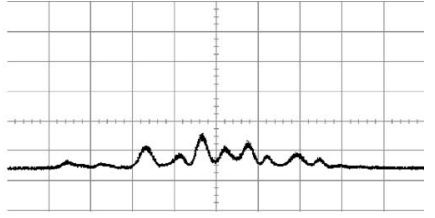

(b)

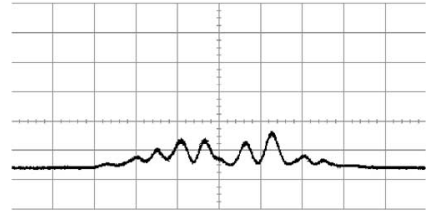

(d)
Fig. 4. Oscilloscope traces when using 25-ps input pulses for (a) autocorrelation, F22:F22*; (b) cross-correlation, F22:F12*; (c) autocorrelation, F22:R22*; (d) cross-correlation, F22:R12* (time scale: $200 \mathrm{ps} / \mathrm{div}$ ).

\section{Correlation Properties}

Direct-sequence OCDMA encoding using the grating devices described earlier is performed by applying a short pulse at the input of the encoding grating. Provided that the pulse is much shorter than the chip length of the grating, and that light can penetrate the full grating length, the reflected signal will follow closely the code superstructure. In the limit that the incoming pulses are long enough, so that their bandwidth is comparable to the reflectivity bandwidth of the grating, any rapidly varying feature on the codes experiences stronger filtering, thereby compromising the shape of the coded waveform. Thus, although the use of longer pulses is more desirable both in terms of cost, efficiency, and ease of generation, increasing the pulse duration has significant consequences for the chip rate, and, subsequently, the code length and/or the strength of the grating.

We tested the correlation properties for each individual grating before undertaking the system experiments. In order to ensure that all features of the encoded sequences were generated reliably after reflection off the gratings, we used 25-ps input pulses, i.e., pulses with a width comparable to the chip length of the codes.

Fig. 4 shows example oscilloscope traces of the autocorrelation and cross correlation, for both the continuous phase-shift and the code-reconfigurable gratings. For all the grating pairs, distinctive autocorrelation pulses and relative low-level crosscorrelation pulses between codes were successfully achieved. As shown, both types of gratings display similar correlation properties due to the excellent match of the phase distribution within the gratings. In both cases, the width of the autocorrelation is $\sim 50$ ps.

\section{TRANSMitTER DESIGN}

In our OCDM/DWDM experiments, the pulsed signal was generated by coupling continuous wave WDM sources into a pulse carving electroabsorption modulator (EAM), driven by a high-frequency radio frequency (RF) signal, as shown in Fig. 5. The generated pulse width could be modified by adjusting the frequency of the RF signal and the bias voltage applied to 


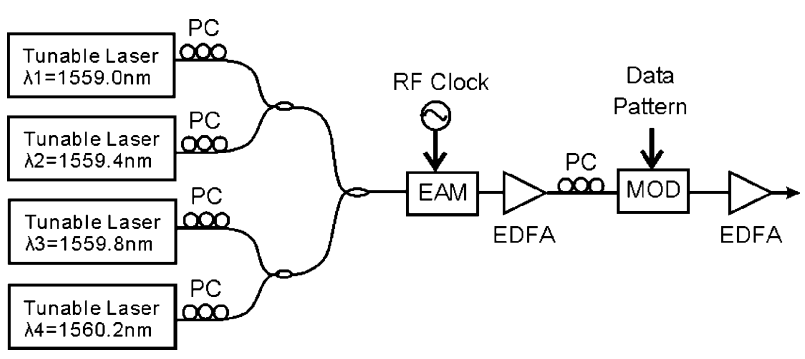

Fig. 5. Experimental setup for DWDM short-pulse generation.

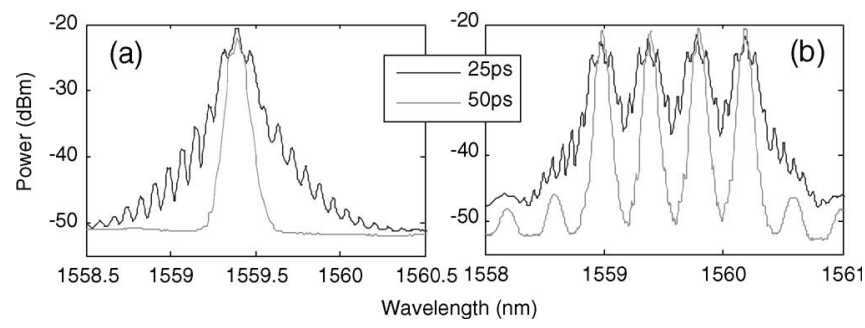

Fig. 6. Measured optical spectra for the 25-ps and 50-ps pulses. (a) Single wavelength channel. (b) Four DWDM channels.

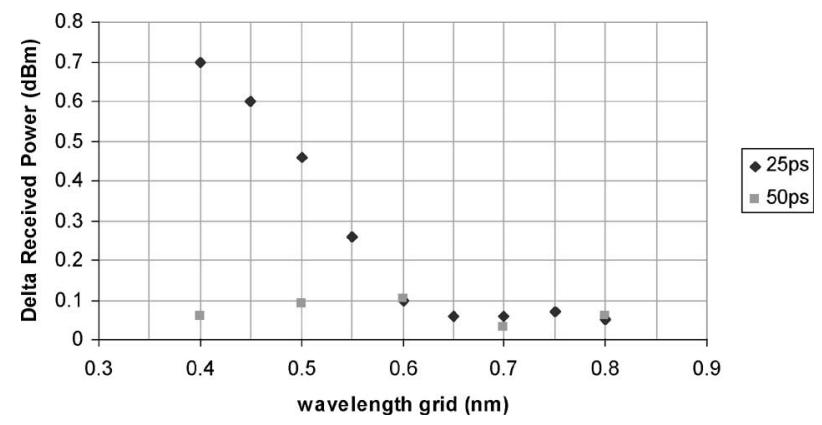

Fig. 7. Received optical power penalty compared to single channel $\left(\lambda_{2}\right.$ only $)$ decoding as a function of the wavelength spacing between adjacent channels in the presence of two interfering WDM channels $\left(\lambda_{1}\right.$ and $\left.\lambda_{3}\right)$.

the EAM. The short pulse trains were then modulated using a $\mathrm{LiNbO}_{3}$ intensity modulator to gate down the repetition rate and/or generate a data pattern.

Thus, by driving the EAM with a $10-\mathrm{GHz}$ RF signal, we were able to generate 25-ps pulses, providing a good match with the chip duration of the encoding SSFBGs. However, from an observation of the resulting optical spectrum, it is clear that their bandwidth is too broad to allow DWDM operation on a $50-\mathrm{GHz}$ channel spacing (see Fig. 6). As shown in Fig. 6(b), the extinction ratio between adjacent channels on a $50-\mathrm{GHz}$ grid was just $\sim 15 \mathrm{~dB}$, implying that there would be significant interchannel crosstalk introduced during the encoding process. This was also confirmed through measurements of the relative power penalty introduced when detecting one OCDMA signal (at wavelength $\left.\lambda_{2}\right)$ in the presence of two adjacent interfering encoded WDM channels (at wavelengths $\lambda_{1}$ and $\lambda_{3}$ ) as a function of the channel spacing. This measurement is presented in Fig. 7, where it is shown that it is not possible to reduce the channel spacing below $0.6 \mathrm{~nm}$ without introducing a significant power penalty.

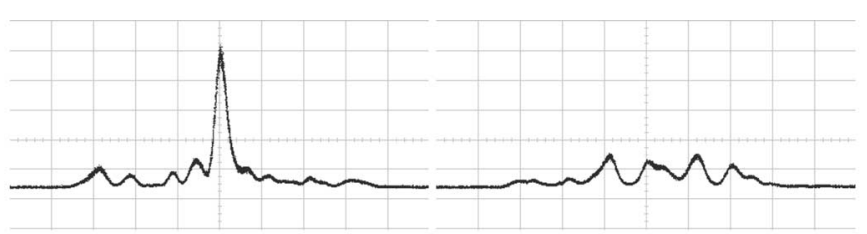

(a)

(b)

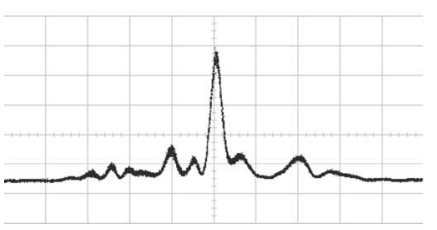

(c)

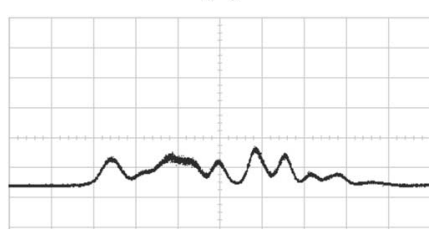

(d)
Fig. 8. Oscilloscope traces when using 50-ps input pulses for (a) autocorrelation, F22:F22*; (b) cross-correlation, F22:F12*; (c) autocorrelation, F22:R22*; (d) cross-correlation, F22:R12* (time scale: 200 ps/div).

We, therefore, investigated the option of using broader pulses at the output of the EAM. By driving the EAM with a $5-\mathrm{GHz}$ signal, we generated 50-ps pulses with a spectrum as shown in Fig. 6 (dashed lines). An extinction ratio of $\sim 30 \mathrm{~dB}$ between adjacent channels was now achieved, and no additional power penalty was introduced when reducing the WDM channel spacing to $0.4 \mathrm{~nm}$ (i.e., $50 \mathrm{GHz}$; see Fig. 7).

In a previous study, we established that the continuous phaseshift SSFBG is more tolerant to the use of wider input pulses compared to a SSFBG incorporating discrete phase shifts [10]. Our simulations also show that these gratings perform well as far as the extinction of the cross-correlation signals is concerned for 50-ps pulses, i.e., a pulse width that is twice the chip duration. Our individual grating characterization experiments confirmed this point. Fig. 8 shows typical oscilloscope traces of autocorrelation and cross-correlation signals, for both the continuous phase-shift and the code-reconfigurable gratings, with an input pulse width of $50 \mathrm{ps}$. These traces can be compared directly to those presented in Fig. 4 for 25-ps input pulses, and exhibit an autocorrelation width of $\sim 60 \mathrm{ps}$.

As can be seen from Figs. 4 and 8, the autocorrelations with 50-ps input pulses still exhibit distinctive correlation properties. The autocorrelation width is slightly wider than for the case of 25-ps input pulses as predicted by theory, which shows that when the input pulse is far wider than the chip duration, the autocorrelation width will be determined by the input pulse.

\section{OCDMA/DWDM SYSTEM EXPERIMENT}

The schematic in Fig. 9(a) shows the experimental configuration of our 16-channel OCDMA/DWDM encoder and decoder system. The incoming signal was formed as described in the previous section. The $\mathrm{LiNbO}_{3}$ intensity modulator shown in Fig. 5 was used to modulate the multiwavelength pulsed signal, thereby generating a $2^{7}-1$ PRBS at $625 \mathrm{Mb} / \mathrm{s}$ at each of the four wavelengths. The modulated pulses were fed to an array of 16 continuous phase-shift SSFBGs to generate data from an effective 16 users. The grating array was composed of four different codes (F1-F4), each code being repeated on each of the 

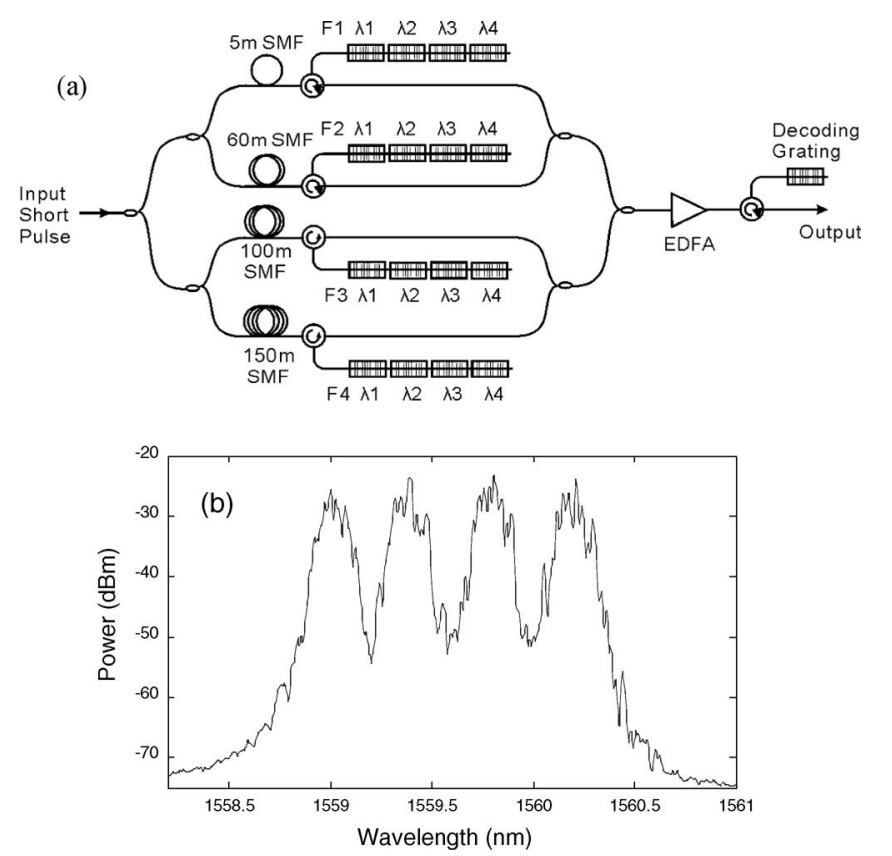

Fig. 9. (a) Experimental 16-channel OCDMA/DWDM system setup. (b) Measured optical spectrum for the encoded 16-channel OCDMA/DWDM signal.

four different central wavelengths $\left(\lambda_{1}-\lambda_{4}\right)$. The SSFBGs were grouped together by their code, thus forming the four branches shown in Fig. 9(a). Different lengths of single-mode fiber (SMF) were included in each branch to ensure that the recombined signals at the output of the encoding system were not mutually coherent. Note that there was no active control over the temporal positioning of the various encoded waveforms. However, the duty cycle of the encoded signals at $625 \mathrm{Mb} / \mathrm{s}$ was around $1: 2$, implying that there was significant overlap between the coded signals at the output of the transmitter. Fig. 9(b) shows the spectrum of the 16-channel encoded signal after being combined into a single fiber. At the receiver end, we used either a (fixed) continuous phase-shift SSFBG or a code-reconfigurable grating to decode a particular target data channel. The inherent wavelength selectivity of the grating is used to filter out the 12 out-of-band channels (four codes at each wavelength). Of the remaining four in-band channels, only the code matched to the decoding grating generates a distinct autocorrelation peak, while the other three unmatched channels produce relatively low-level cross-correlation signals.

Fig. 10(a) and (c) show the result of decoding F33 by using fixed-coded SSFBG F33* and reconfigurable grating R33*, respectively, when only code F3 (at all four wavelengths) was present in the incoming signal. The clear eye diagrams highlight the excellent wavelength discrimination provided by the continuous phase-shift decoders. Fig. 10(b) and (d) show the results of decoding input channel F33, using fixed-coded grating F33* and reconfigurable grating R33*, respectively, when all the 16 channels were incident on the receiver. The eye diagrams in Fig. 10(b) and (d) are somewhat degraded relative to those in Fig. 10(a) and (c) due to multiple access interference (MAI) noise from the other in-band channels. However, an open eye

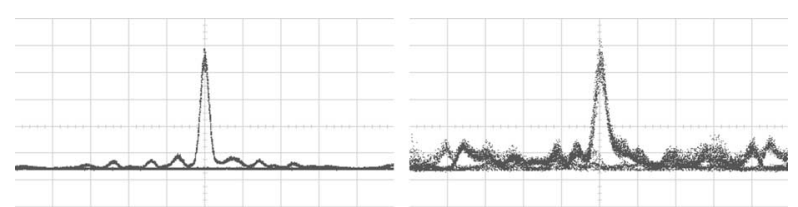

(a)

(b)

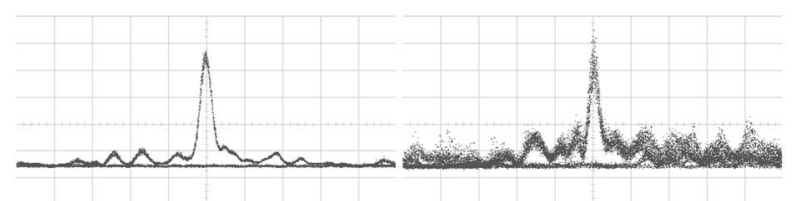

(c)

(d)

Fig. 10. Oscilloscope traces (time scale: $200 \mathrm{ps} / \mathrm{div}$ ) for (a) using F33* and (c) using R33* to decode F33 when only F3 at four wavelengths was present in the incoming signal, (b) using F33* and (d) using R33* to decode F33 when all 16 channels were present.
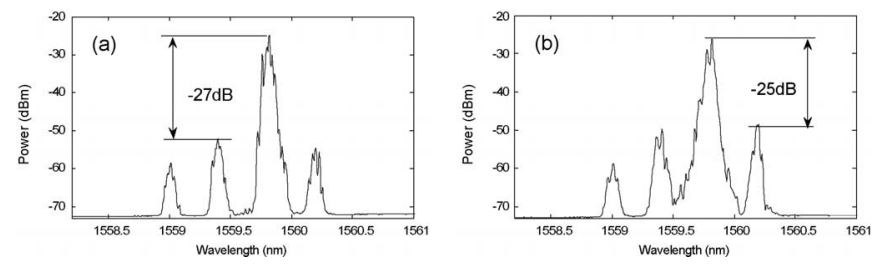

Fig. 11. (a) and (b). Corresponding optical spectra for Fig. 10 (b) and (d), respectively.

is still achieved and the performance of both fixed and reconfigurable gratings is seen to be comparable. The corresponding received optical spectra are shown in Fig. 11. The interchannel spectral extinction ratio is better than $-25 \mathrm{~dB}$ in all cases.

We evaluated the noise performance of the system through a series of bit-error rate (BER) measurements, which are summarized in Fig. 12. In Fig. 12(a), we plot the BER curves obtained using decoder grating F33*. First, code F33 was transmitted alone, and, then, in the presence of other wavelength channels (each transmitting the same OCDMA code F3). Finally, we progressively added more OCDMA codes into the system (codes $\mathrm{F} 1, \mathrm{~F} 2$, and F4, respectively). The figure shows that when there is only one OCDMA code (F33) in the incoming signal, the effect of the out-of-band channels is negligible. A power penalty of $\sim 2.1 \mathrm{~dB}$ is associated with the OCDMA decoding of this signal, whereas the presence of the remaining DWDM channels introduces no further penalty when used at a $50-\mathrm{GHz}$ grid spacing. With all four DWDM wavelengths turned on, the additional codes are added, resulting in a corresponding rise in the power penalty due to the increasing MAI. $\sim 1.5-\mathrm{dB}$ power penalty for each additional in-band code was measured at a BER of $10^{-9}$; however, error-free performance was achieved in all instances.

Fig. 12(b) is shown to facilitate a comparison between the fixed and reconfigurable decoders. In this case, we study the performance of four different channels, namely F22, F23, F32, and F33. For the assessment of the fixed decoders, a different SSFBG is used to decode each of the channels, whereas obviously for the reconfigurable decoder, the same device was tuned to decode the various incoming signals. We first measured the noise performance of the system when just one OCDMA code per wavelength channel was transmitted, and then repeated 

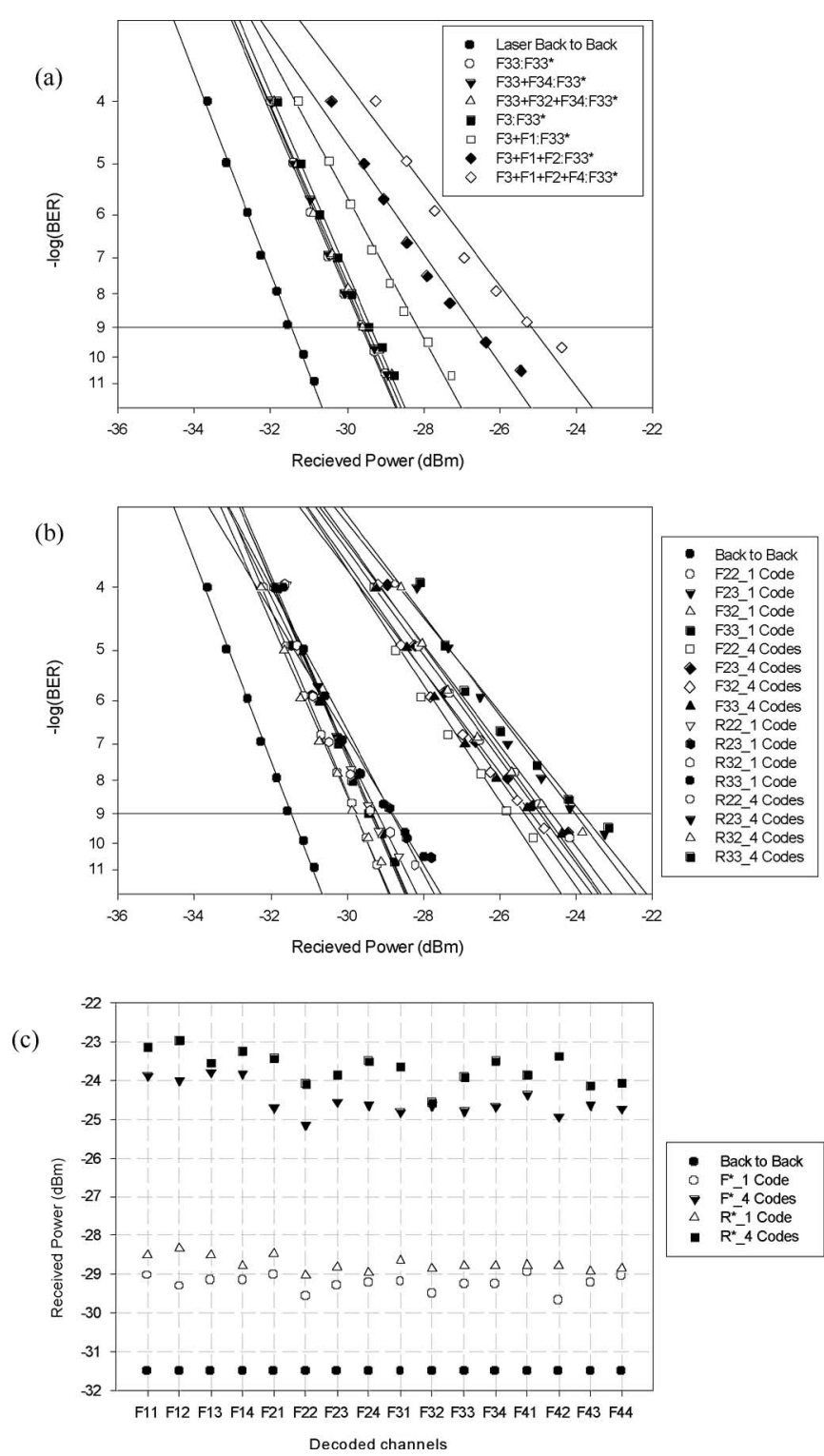

Fig. 12. (a) BER results for decoding F33, with increasing interference channels. (b) BER results for decoding F22, F23, F32, F33, with 1- and 4 in-band codes $\times 4$ DWDM channels. (c) Received optical power for decoding each of the 16 channels at a BER $=10^{-9}$.

the measurements in the presence of all 16 channels. As before, the noise degradation is predominantly associated with the inclusion of more OCDMA codes. However, error-free performance is achieved for all channels tested. In agreement with the trend shown in Fig. 12(a), inclusion of four in-band codes (16 channels in total) is associated with an additional $\sim 4.5-\mathrm{dB}$ power penalty (at BER $=10^{-9}$ ) relative to the case when only one OCDMA code is present (four wavelength channels). The performance of the reconfigurable decoder is seen to be only slightly worse than that of the fixed-code decoder. Note that some form of nonlinear thresholding technique at the receiver could be used to improve the performance albeit at an additional cost [14].

We have only presented detailed BER measurements at $\lambda_{2}$ and $\lambda_{3}$ since these are the most vulnerable to interchannel crosstalk.
However, a summary of the performance of all codes both in the absence of any OCDMA-induced MAI and under full 16channel operation is presented in Fig. 12(c). This figure shows the power level required at the receiver in order to achieve a $10^{-9}$ BER, and highlights that uniform performance could be achieved for all 16 channels. In agreement to the results shown in Fig. 12(b), the power penalty between the fixed-coded decoder and the reconfigurable decoder is $\sim 0.5 \mathrm{~dB}$ when only one OCDMA channel is present, which increases to $\sim 1 \mathrm{~dB}$ in the presence of all four OCDMA channels. We believe that improvements in the thermal stability of the reconfigurable device will help improve its performance relative to the fixed decoders. Finally, we attribute the small variations in the received power required to achieve error-free operation for the various codes, to be primarily due to slight differences in the correlation properties of the coding sequences. However, the relative timing (which was set arbitrarily in this experiment) of the various transmitted OCDMA code sequences might also contribute to this variation.

\section{CONCLUSION}

We have experimentally demonstrated a 16-channel OCDMA/DWDM system based on new SSFBG-based coding/decoding approaches. The narrow bandwidth of continuous phase-shift SSFBGs allows the DWDM grid spacing to be reduced down to $50 \mathrm{GHz}$. We have demonstrated reconfigurability at the receiver by employing a dynamically addressable decoder, and have shown that no significant additional power penalty is associated with its use relative to a fixed-code decoder. Relatively wide input pulses of $\sim 50$-ps duration (twice the chip duration) were employed in the system to allow use of 50-GHz DWDM channel spacing, confirming the capability of both the continuous phase-shift SSFBG and reconfigurable grating technologies to tolerate wider input pulses without significant degradation in their correlation properties. We have demonstrated that error-free performance was achieved for all 16 channels. Furthermore, it was shown that most significant power penalties were introduced from the correlation process when decoding OCDMA signals at a given wavelength, whereas wavelength filtering of the DWDM channels did not introduce any significant additional penalty. One can, therefore, envisage that a system like the one described herein can be directly scaled by extending its wavelength dimension. It is also clear that DS-OCDMA based on SSFBG coding techniques is fully compatible with WDM technologies, allowing, for example, OCDMA wavelength channels to coexist with other WDM channels carrying conventionally encoded data.

\section{REFERENCES}

[1] P. C. Teh, P. Petropoulos, M. Ibsen, and D. J. Richardson, “A comparative study of the performance of seven- and 63-chip optical code-division multiple-access encoders and decoders based on superstructured fiber Bragg gratings," J. Lightwave. Technol., vol. 19, no. 9, pp. 1352-1365, Sep. 2001.

[2] P. C. Teh, P. Petropoulos, M. Ibsen, and D. J. Richardson, "Phase encoding and decoding of short pulses at $10 \mathrm{~Gb}$," IEEE Photon. Technol. Lett., vol. 13 , no. 2 , pp. 154-156, Feb. 2001. 
[3] P. C. Teh, M. Ibsen, J. H. Lee, P. Petropoulos, and D. J. Richardson, "A 4-channel WDM/OCDMA system incorporating 255-chip 320 Gchip/s quaternary phase coding and decoding gratings," in Proc. Opt. Fiber Commun. Conf. Exhib. 2001, vol. 4, pp. PD37-1-PD37-3.

[4] P. C. Teh, M. Ibsen, L. B. Fu, J. H. Lee, Z. Yusoff, and D. J. Richardson, "A 16-channel OCDMA system (4 OCDM r 4 WDM) based on 16chip, $20 \mathrm{Gchip} / \mathrm{s}$ superstructure fibre Bragg gratings and DFB fibre laser transmitters," in Proc. Opt. Fiber Commun. Conf. Exhib. 2002, Anaheim, CA, pp. 600-601.

[5] M. R. Mokhtar, M. Ibsen, P. C. Teh, and D. J. Richardson, "Reconfigurable multilevel phase-shift keying encoder-decoder for all-optical networks," IEEE Photon. Technol. Lett., vol. 15, no. 3, pp. 431-433, Mar. 2003.

[6] Z. Zhang, C. Tian, M. R. Mokhtar, P. Petropoulos, D. J. Richardson, and M. Ibsen, "Rapidly reconfigurable optical phase encoder-decoderss based on fiber Bragg gratings," IEEE Photon. Technol. Lett., vol. 18, no. 11, pp. 1216-1218, Jun. 2006

[7] C. Dorrer and I. Kang, "Simultaneous temporal characterization of telecommunication optical pulses and modulators by use of spectrograms," Opt. Lett., vol. 27, pp. 1315-1317, 2002.

[8] Z. Zhang, C. Tian, M. A. F. Roelens, M. R. Mokhtar, P. Petropoulos, D. J. Richardson, and M. Ibsen, "Direct characterization of the spatial effective refractive index profile in Bragg gratings," IEEE Photon. Technol. Lett., vol. 17, no. 12, pp. 2685-2687, Dec. 2005.

[9] C. Tian, Z. Zhang, M. Ibsen, P. Petropoulos, and D. J. Richardson, "A reconfigurable optical header recognition system for optical packet routing applications," IEEE Photon. Technol.Lett., vol. 18, no. 22, pp. 2395-2397, Nov. 2006.

[10] Z. Zhang, C. Tian, P. Petropoulos, D. J. Richardson, and M. Ibsen, "Distributed-phase OCDMA encoder-decoders based on fiber Bragg gratings," IEEE Photon. Technol. Lett., vol. 19, no. 8, pp. 574-576, Apr. 15, 2007.

[11] J. Canning and M. G. Sceats, "p phase-shifted periodic distributed structures in optical fibres by UV post-processing," Electron. Lett., vol. 30, pp. 1344-1345, 1994.

[12] D. Uttamchandani and A. Othonos, "Phase shifted Bragg gratings formed in optical fibres by post-fabrication thermal processing," Opt. Commun. vol. 127, pp. 200-204, 1996.

[13] S. Boztas, R. Hammons, and P. Y. Kumar, "4-phase sequences with nearoptimum correlation properties," IEEE Trans. Inf. Theory, vol. 38, no. 3 , pp. 1101-1113, May 1992.

[14] J. H. Lee, P. C. Teh, P. Petropoulos, M. Ibsen, and D. J. Richardson, "A grating-based OCDMA coding-decoding system incorporating a nonlinear optical loop mirror for improved code recognition and noise reduction," J. Lightw. Technol., vol. 20, no. 1, pp. 36-46, Jan. 2002.

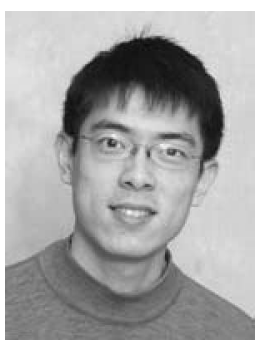

Chun Tian received the B.S. degree in telecommunications engineering from Beijing University of Posts and Telecommunications, Beijing, China, in 2000.

From 2000 to 2003, he was an R\&D Engineer at Datang Telecom Technology Company Ltd., Beijing. Since 2003, he has been with the Optoelectronics Research Centre, University of Southampton, Southampton, U.K., where he is currently a Research Scholar. His current research interests include OCDMA and optical packet switching systems.

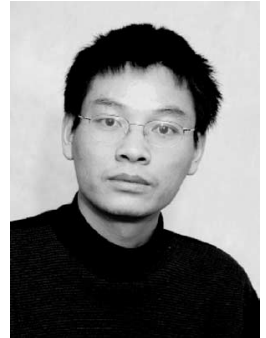

Zhaowei Zhang was born in Hubei, China. He received the Graduate degree from the Department of Optoelectronics Engineering, Huazhong University of Science and Technology, Wuhan, China, in 1998, and the M. Sc. degree in optics from the Department of Applied Physics, Shanghai Jiao Tong University, Shanghai, China, in 2001. He is currently working toward the Ph.D. degree at the Optoelectronics Research Centre, University of Southampton, Southampton, U.K.

His current research interests include fiber Bragg gratings and high-power fiber lasers.

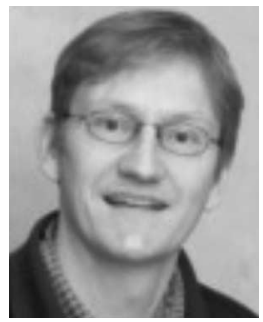

Morten Ibsen was born in Copenhagen, Denmark. He received the Graduate degrees in physics, mathematics, and optical communications from the IFAUniversity of Aarhus, Aarhus, Denmark, OFTCUniversity of Sydney, Australia, and the Optoelectronics Research Centre-University of Southampton, Southampton, U.K., respectively.

$\mathrm{He}$ is currently a Research Scholar on a university research fellowship from the Royal Society of London. His current research interests include specialized Bragg grating design, fabrication, and characterization; and dynamic aspects of Bragg gratings including pulse shaping and tunable dispersion equalization together with devices and systems utilizing Bragg gratings.

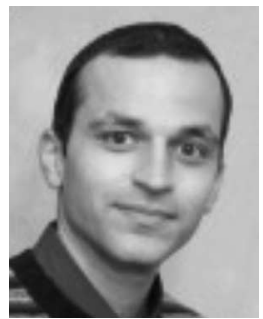

Periklis Petropoulos was born in Patras, Greece. He received the Graduate degree from the Department of Electrical Engineering and Information Technology, University of Patras, Patras, in 1995, the M.Sc. degree in communications engineering from the University of Manchester Institute of Science and Technology, Manchester, U.K., in 1996, and the Ph.D. degree in optical telecommunications from the Optoelectronics Research Centre (ORC), University of Southampton, Southampton, U.K., in 2000.

Since 2000 , he has been with the ORC, where he is currently a Reader. He is the author or coauthor of more than 160 papers published in international journals in the field of optical physics and optical communications. His current research interests include all-optical processing and switching in optical fibers; pulse manipulation for optical communications using fiber Bragg gratings, including applications in optical correlation systems for the implementation of optical code-division multiple access and optical packet switched systems; silica and compound glass holey fibers and their nonlinear applications; and fiber lasers.

Dr. Petropoulos is a member of the Optical Society of America.

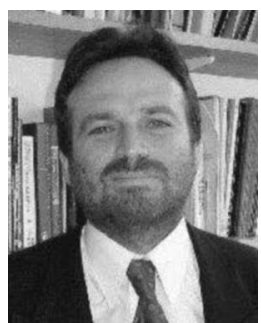

David J. Richardson was born in Southampton, U.K., in 1964. He received the B.Sc. and Ph.D. degrees in fundamental physics from Sussex University, Brighton, U.K., in 1985 and 1989, respectively.

In 1989, he joined the Optoelectronics Research Centre, University of Southampton, Southampton, U.K. where he is now a Deputy Director. He is the author or coauthor of more than 550 papers published in international journals. He is the holder of over 20 patents. He is one of the Cofounders of Southampton Photonics Inc., Southampton, U.K. His current research interests include holey fibers, high-power fiber lasers, short pulse generation, nonlinear optics, and high-capacity optical communication systems. 